\title{
The Extremes of the Underlying Event
}

\author{
T. Martin ${ }^{a *}$, P. Skands ${ }^{b}$, S. Farrington ${ }^{a}$ \\ ${ }^{a}$ University of Warwick, UK \\ ${ }^{a}$ Monash University, Australia \\ E-mail: Tim.Martin@cern.ch
}

\begin{abstract}
Our understanding of the transition from partons to hadrons in QCD has been challenged in recent years. In particular for high-multiplicity minimum-bias events at the LHC, novel experimentation techniques have revealed tantalising indications of non-trivial and possibly collective phenomena. A new wave of model building efforts has ensued, including ideas of colour reconnections, colour ropes, interacting strings, and hydrodynamics. It is essential to develop further sensitive probes of the mechanisms underpinning this fundamental aspect of QCD, to find answers to key questions such as if and how jet universality is broken and whether collective effects are relevant in pp collisions. The answers will not only be of fundamental interest but will also impact precision studies that rely on hadronisation models, as well as the interpretation of heavy-ion results that use pp collisions as a reference. Using strangeness and baryons as tracers, we propose an extension of minimum-bias studies adapted to the environment of the underlying event which show substantial discriminatory power between several alternative models of soft physics.
\end{abstract}

38th International Conference on High Energy Physics

3-10 August 2016

Chicago, USA

${ }^{*}$ Speaker. 


\section{The Underlying Event}

A brief summary of the work in [1] is presented. The underlying event (UE, see [2]) encompasses the collection of final state particles produced from a single hadronic (here, $p p$ ) collision whose origin is not directly related to the 'hardest' parton-parton scattering in the event, but whose make-up is dominated instead by multiple soft parton interactions (MPI). Activity levels in the underlying event are typically measured in regions of the azimuth with respect to a leading transverse object $\left(p_{\mathrm{T}}^{\text {lead }}\right)$ which plays the role of a transverse thrust axis for the event. The UE 'transverse region', spanning the azimuthal region $90^{\circ} \pm 30^{\circ}$ with respect to the $p_{\mathrm{T}}^{\text {lead }}$ object, receives minimal contributions from the hard scatter and any associated recoil. Mean activity levels ${ }^{1}$ in this 'transverse region' are therefore sensitive to the average amount of MPI as a function of $p_{\mathrm{T}}^{\text {lead }}$. For leading track-jet (anti- $k_{t} R=0.4$ [3]) studies, it is observed that the average activity level in the transverse region rises as a function of $p_{\mathrm{T}}^{\text {lead }}$. This rise is correlated with a shrinking $p p$ impact parameter such that for $p_{\mathrm{T}}^{\text {lead }}>10 \mathrm{GeV}$, the impact parameter is small and the average activity level in the transverse region plateaus with only a small residual dependence on $p_{\mathrm{T}}^{\text {lead }}$, driven by wide-angle radiation from the hard scatter [4].

\section{The Extremes of the Underlying Event}

While the mean activity level, $\langle N($ Trans. $)\rangle$, in the UE transverse region reaches a plateau, there are large event-by-event fluctuations correlated to the number of MPI in each event. We define the transverse ratio variable $R_{T}=N$ (Trans.) $/\langle N($ Trans. $)\rangle$ for events satisfying $10<p_{\mathrm{T}}^{\text {lead }}<30 \mathrm{GeV}$. $R_{T}$ relates the per-event transverse activity level to the average over all events in the sample. For $R_{T} \ll 1$, MPI accompanying the hard scatter is suppressed and LEP-style fragmentation behaviour is expected. For $R_{T}>1$, a large number of MPI is expected and their correlations within the colourfield of the protons can give rise to collective phenomena which must be accounted for in order to describe data. Three models which attempt to describe this high-MPI region in differing ways are Pythia 8.2 with a new colour-reconnection model, Dipsy with colour ropes and Epos. In brief, Pythia 8.2 with the Monash tune [5, 6, 7] introduces a new colour re-connection model which approximates to the full group theoretical weights from SU(3) and allows for three-way 'string junctions' to form [8]. Note that a further set of models including thermal and close-packing effects were implemented in Pythia 8.2 after this work was completed, see [9]. Dipsy [10] allows for geometrically nearby strings to act coherently as a 'colour rope' with higher intrinsic tension which affects their hadronisation [11]. Epos [12, 13] identifies high-density 'core' regions of the event which are subject to a hydrodynamic modelling. The effects of these differing modelling choices are investigated using $p p$ Monte Carlo simulation at $\sqrt{s}=13 \mathrm{TeV}$.

\section{Baryons and Strange Hadrons as Hadronisation Probes}

Baryon production requires an antisymmetric combination of three colours which makes it potentially sensitive to sub-leading colour correlations between nearby partons while the relative suppression of strange quarks is particularly sensitive to the effective scale of hadronisation since

\footnotetext{
${ }^{1}$ Here $\langle N($ Trans. $)\rangle$, the number of particles in the transverse region where the average is over all events.
} 
$m_{s} \sim \Lambda_{Q C D}$. Baryon and strange-hadron multiplicities are hence useful experimental probes when investigating the hadronisation potential in the UE as a function of $R_{T}$.

In Fig. 1(a), the ratio of the average $N(p+\bar{p})$ to the average inclusive $N$ particle multiplicity in the transverse region is plotted as a function of $R_{T}$. For the more advanced colour-reconnection in Pythia (New CR) and Dipsy Rope, a $\sim 40 \%$ larger normalised proton yield is predicted with respect to the baseline Pythia and Dipsy NoSwing models. The more advanced models additionally show a greater dependence on $R_{T}$ than the baseline models as observed from the trend in the ratio plot. Epos has the overall strongest trend with $R_{T}$ but predicts a smaller normalised proton yield than the Pythia New CR and Dipsy Rope models.

In Fig. 1(b), the ratio of the average Lambda baryon to average $K_{S}^{0}$ meson multiplicity in the transverse region is plotted as a function of $R_{T}$. Here the baseline Pythia and Dipsy NoSwing models predict little to no dependence on $R_{T}$ whereas the more advanced models: Pythia New CR, Dipsy Rope and Epos all show a strong dependence resulting in higher relative Lambda baryon yields at large values of $R_{T}$, reaching a $90 \%$ enhancement by $R_{T}=4$
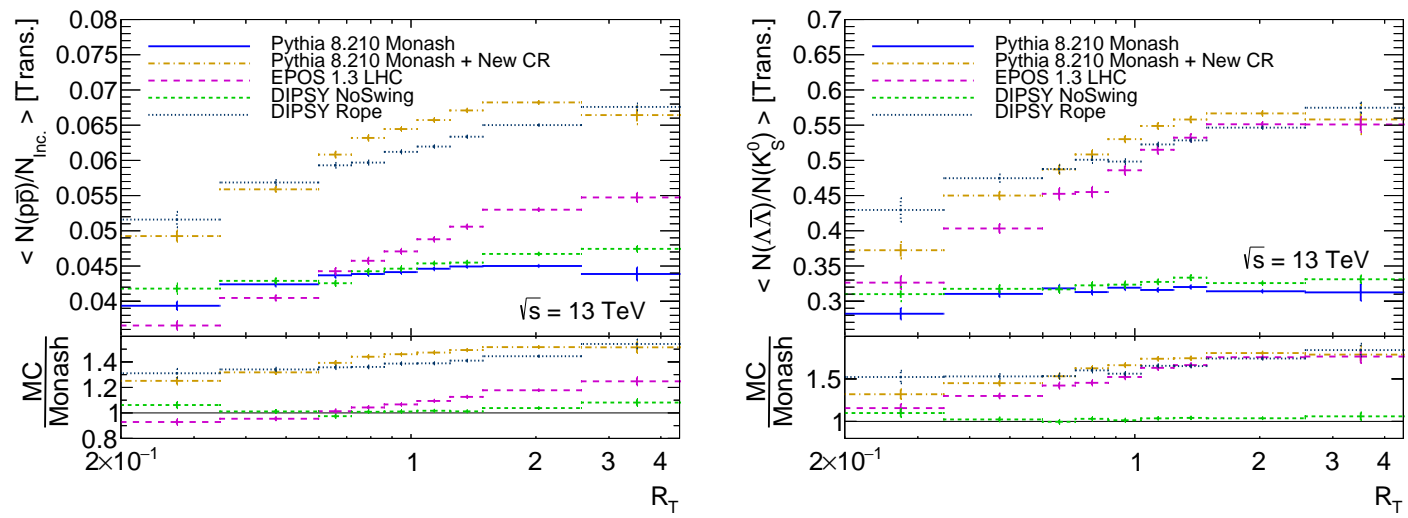

Figure 1: Ratio of average $p+\bar{p}$ multiplicity to inclusive yield (a) and $\Lambda+\bar{\Lambda}$ to $K_{S}^{0}$ multiplicity (b). Both as a function of the per-event inclusive transverse multiplicity with respect to its mean value. The inclusive yield contains charged stable particles along with identified strange hadrons, cuts on $p_{\mathrm{T}}>200 \mathrm{MeV}$ and $|\eta|<2.5$ were applied to all particles.

\section{Conclusion}

The multiplicity of strange hadrons and baryons are shown in Monte Carlo to be effective discriminants against different modelling of the hadronisation potential in the 'transverse region' of the underlying event as a function of the the per-event activity level in the underlying event with respect to the mean. When measured in data, these variables will allow for discrimination between competing models of hadronisation in high-multiplicity proton proton interactions where collective phenomena are expected to become important. It will be particularly interesting to determine if the trends in the UE match those recently observed by the ALICE collaboration in high-multiplicity minimum-bias events [14]. 


\section{References}

[1] T. Martin, P. Skands and S. Farrington, Eur. Phys. J. C 76 (2016) no.5, 299 doi:10.1140/epjc/s10052-016-4135-4 [arXiv:1603.05298 [hep-ph]].

[2] T. Affolder et al. [CDF Collaboration], Phys. Rev. D 65 (2002) 092002. doi:10.1103/PhysRevD.65.092002

[3] M. Cacciari, G. P. Salam and G. Soyez, JHEP 0804 (2008) 063 doi:10.1088/1126-6708/2008/04/063 [arXiv:0802.1189 [hep-ph]].

[4] G. Aad et al. [ATLAS Collaboration], Phys. Rev. D 86 (2012) 072004 doi:10.1103/PhysRevD.86.072004 [arXiv:1208.0563 [hep-ex]].

[5] T. Sjöstrand, S. Mrenna and P. Z. Skands, JHEP 0605 (2006) 026 doi:10.1088/1126-6708/2006/05/026 [hep-ph/0603175].

[6] T. Sjöstrand et al., Comput. Phys. Commun. 191 (2015) 159 doi:10.1016/j.cpc.2015.01.024 [arXiv:1410.3012 [hep-ph]].

[7] P. Skands, S. Carrazza and J. Rojo, Eur. Phys. J. C 74 (2014) no.8, 3024 doi:10.1140/epjc/s10052-014-3024-y [arXiv:1404.5630 [hep-ph]].

[8] J. R. Christiansen and P. Z. Skands, JHEP 1508 (2015) 003 doi:10.1007/JHEP08(2015)003 [arXiv:1505.01681 [hep-ph]].

[9] N. Fischer and T. Sjöstrand, arXiv:1610.09818 [hep-ph].

[10] C. Flensburg, G. Gustafson and L. Lönnblad, JHEP 1108 (2011) 103 doi:10.1007/JHEP08(2011)103 [arXiv:1103.4321 [hep-ph]].

[11] C. Bierlich, G. Gustafson, L. Lönnblad and A. Tarasov, JHEP 1503 (2015) 148 doi:10.1007/JHEP03(2015)148 [arXiv:1412.6259 [hep-ph]].

[12] K. Werner, F. M. Liu and T. Pierog, Phys. Rev. C 74 (2006) 044902 doi:10.1103/PhysRevC.74.044902 [hep-ph/0506232].

[13] T. Pierog, I. Karpenko, J. M. Katzy, E. Yatsenko and K. Werner, Phys. Rev. C 92 (2015) no.3, 034906 doi:10.1103/PhysRevC.92.034906 [arXiv:1306.0121 [hep-ph]].

[14] J. Adam et al. [ALICE Collaboration], arXiv:1606.07424 [nucl-ex]. 